\title{
Enhancing Methotrexate Tolerance with Folate Tagged Liposomes in Arthritic Mice
}

\author{
Eugénia Nogueira ${ }^{1,2}$, Franck Lager ${ }^{3,4,5}$, Delphine Le Roux ${ }^{3,4,5}$, Patrícia Nogueira ${ }^{6,7}$, Jaime Freitas ${ }^{6,7}$, \\ Celine Charvet ${ }^{3,4,5}$, Gilles Renault ${ }^{3,4,5}$, Ana Loureiro, ${ }^{1,2}$, Catarina R. Almeida ${ }^{8}$, Anna Ohradanova-Repic ${ }^{9}$, \\ Christian Machacek ${ }^{9}$, Gonçalo J. L. Bernardes ${ }^{10,11}$, Alexandra Moreira ${ }^{6,7}$, Hannes Stockinger ${ }^{9}$, \\ Michael Burnet ${ }^{12}$, Alexandre M. Carmo ${ }^{6,7,13}$, Andreia C. Gomes ${ }^{1}$, Ana Preto ${ }^{1}$, \\ Georges Bismuth ${ }^{3,4,5}$, and Artur Cavaco-Paulo ${ }^{2, *}$ \\ ${ }^{1}$ CBMA-Centre of Molecular and Environmental Biology, Department of Biology, University of Minho, \\ Campus of Gualtar, 4710-057, Braga, Portugal \\ ${ }^{2}$ CEB - Centre of Biological Engineering, University of Minho, Campus of Gualtar, 4710-057, Braga, Portugal \\ 3 INSERM-Institut National de la Santé et de la Recherche Médicale, U1016, Institut Cochin, Paris, France \\ ${ }^{4}$ Université Paris Descartes, 12 Rue de l'Écolede Médecine, 75006 Paris, France \\ ${ }^{5}$ CNRS - Centre National de la Recherche Scientifique, UMR 8104, Paris, France \\ ${ }^{6}$ Instituto de Investigação e Inovação em Saúde, Universidade do Porto, 4150-180, Portugal \\ ${ }^{7}$ IBMC - Instituto de Biologia Molecular e Celular, Rua do Campo Alegre 823, 4150-180, Porto, Portugal \\ 8 INEB — Instituto de Engenharia Biomédica, NEWTherapies Group, Universidade do Porto, Rua do Campo Alegre 823, \\ 4150-180, Porto, Portugal \\ ${ }^{9}$ Medical University of Vienna, Center for Pathophysiology, Infectiology and Immunology, \\ Institute for Hygiene and Applied Immunology, 1090, Vienna, Austria \\ ${ }^{10}$ Department of Chemistry, University of Cambridge, Lensfield Road, Cambridge, CB2 1EW, United Kingdom \\ ${ }^{11}$ Instituto de Medicina Molecular, Faculdade de Medicina da Universidade de Lisboa, Av. Professor Egas Moniz, \\ 1649-028, Lisboa, Portugal \\ ${ }^{12}$ Synovo GmbH, Paul Ehrlich Street 15, D-72076 Tüebingen, Germany \\ ${ }^{13}$ ICBAS - Instituto de Ciências Biomédicas Abel Salazar, Universidade do Porto, Rua Jorge Viterbo Ferreira, 228, \\ 4050-313, Porto, Portugal
}

\begin{abstract}
Methotrexate is the first line of treatment of rheumatoid arthritis. Since many patients become unresponsive to methotrexate treatment, only very expensive biological therapies are effective and increased methotrexate tolerance strategies need to be identified. Here we propose the encapsulation of methotrexate in a new liposomal formulation using a hydrophobic fragment of surfactant protein conjugated to a linker and folate to enhance their tolerance and efficacy. In this study we aim to evaluate the efficiency of this system to treat rheumatoid arthritis, by targeting folate receptor $\beta$ present at the surface of activated macrophages, key effector cells in this pathology. The specificity of our liposomal formulation to target folate receptor $\beta$ was investigated both in vitro as in vivo using a mouse model of arthritis (collagen-induced arthritis in DBA/1J mice strain). In both systems, the liposomal constructs were shown to be highly specific and efficient in targeting folate receptor $\beta$. These liposomal formulations also significantly increase the clinical benefit of the encapsulated methotrexate in vivo in arthritic mice, together with reduced expression of CD39 and CD73 ectonucleotidases by joint-infiltrating macrophages. Thus, our formulation might be a promising cost effective way to treat rheumatoid arthritis and delay or reduce methotrexate intolerance.
\end{abstract}

KEYWORDS: Rheumatoid Arthritis, Methotrexate, Macrophages, Folate Receptor, Liposomes.

\footnotetext{
*Author to whom correspondence should be addressed

Email: artur@deb.uminho.pt
}

Received: 13 January 2015

Revised/Accepted: 18 March 2015 


\section{INTRODUCTION}

Rheumatoid arthritis (RA) is a common chronic inflammatory and destructive arthropathy that consumes considerable personal, social, and economic costs. ${ }^{1,2}$ It comprises a syndrome of pain, stiffness, and symmetrical synovitis of diarthrodial joints. ${ }^{3}$ Disease-modifying antirheumatic drugs (DMARDs) slow disease progression and can induce disease remission in some patients. Methotrexate (MTX) is the most commonly prescribed DMARD. ${ }^{4,5}$ However, the toxicity associated with low-dose MTX administration is usually mild and self-limiting. Nausea without vomiting and malaise are the most frequent adverse effects of MTX treatment. ${ }^{6,7}$ Other effects include gastrointestinal or bone marrow toxicity, pneumonitis, hepatotoxicity and cirrhosis. $^{8}$

Furthermore, many patients do not respond to MTX treatment. If patients are MTX intolerant another DMARD should be used or added, or alternatively biologic agents. ${ }^{4}$ In RA therapy biological agents used are anti-TNF- $\alpha$ molecules, responsible for the neutralization of TNF- $\alpha$, the master regulator of RA immunopathogenesis. The development of biological substances for the treatment of rheumatic conditions has been accompanied by ongoing health economic discussions regarding the implementation of these highly effective, but accordingly, highly priced drugs in the standard treatment guidelines of rheumatic diseases. ${ }^{9}$ In this way, more efficient strategies have to be identified in order to improve inflammatory disease treatments while decreasing the MTX side effects with an improved cost-benefit ratio.

We previously describe an innovative strategy for targeted liposomal delivery that uses a hydrophobic fragment of surfactant protein D (SP-D) conjugated to a linker and folic acid (FA or folate), the SP-DS3 peptide, more efficient than classic systems (polyethylene glycol, PEG, linker). ${ }^{10}$

The biological evaluation and characterization of folate receptor (FR) $\alpha$ targeting using these novel liposomal constructs demonstrates their capacity to carry and deliver drugs to target cancer cells.

Although the etiology of RA remains controversial, activated macrophages have been identified as key mediators. ${ }^{11,12}$ It is only after activation that macrophages express the functional form of $\operatorname{FR} \beta,{ }^{13,14}$ which binds the vitamin FA with high affinity $\left(K d<10^{-9} \mathrm{M}\right)$. Here, we aim to demonstrate the ability of our delivery system for treatment of RA by targeting this macrophage receptor. The biological evaluation and characterization of FR targeting using these novel liposomal constructs demonstrates their capacity to carry and deliver MTX to target activated macrophages while not affecting normal cells, both in vitro and in vivo. This was demonstrated in a pre-clinical mice model of arthritis leading to a remarkable clinical benefit. The novel tailored liposomes open new clinical avenues for the diagnosis and treatment of human diseases including chronic inflammatory diseases and cancer.

\section{MATERIALS AND METHODS Materials}

1,2-dioleoyl-sn-glycero-3-phosphoethanolamine (DOPE) and $N$-(carbonyl methoxypolyethylene glycol-2000)1,2-distearoyl-sn-glycero-3-phosphoethanolamine (DSPEMPEG) were obtained from Lipoid GmbH (Germany). Cholesterol $(\mathrm{CH})$, fluorescein isothiocyanate (FITC) and MTX were obtained from Sigma (USA). Alexa Fluor 647 was obtained from Invitrogen (USA).

\section{Peptide Synthesis}

The neck-domain peptide constructed from SP-D was synthesized by American Peptide (USA). FA was covalently bound at the $\mathrm{N}$-terminus of the peptides via glutamic acid and pteroic acid. Peptides were purified to greater than $75 \%$ homogeneity, as determined by analytical highperformance liquid chromatography, and characterized by mass spectrometry.

\section{Liposome Preparation}

Liposomes were prepared using a thin film hydration method. The formulations produced are based on DOPE/CH/DSPE-MPEG which may contain FA-peptide for specific targeting and/or FITC/Alexa Fluor 647 for fluorescence labelling and/or MTX as drug (Table I). Briefly, known amounts of DOPE, $\mathrm{CH}$ and DSPE-MPEG were dissolved in chloroform in a $50 \mathrm{~mL}$ round-bottom flask. For macrophage internalization studies, FITC was incorporated in the lipidic film.

The organic solvent was evaporated using a rotary evaporator followed by additional evaporation under reduced pressure by a high-vacuum system to remove remaining traces of chloroform. The resultant dried lipid film was dispersed in phosphate buffered saline (PBS) buffer containing either peptides, Alexa Fluor 647 or the MTX. The mixture was vortex-mixed at a temperature greater than the phase-transition temperature (room temperature) to yield multilamellar vesicles, which were then extruded (extruder supplied by Lipex Biomembranes Inc., Canada) through $200 \mathrm{~nm}$ pore size polycarbonate filters (Nucleopore, USA) followed by several passages through $100 \mathrm{~nm}$ polycarbonate filters (Nucleopore, USA) to form large unilamellar vesicles. The free peptide, Alexa Fluor 647 and the MTX that was not incorporated into liposomes was removed from the samples after passage through a gel filtration chromatography column (GE Healthcare, USA).

Table I. Different liposomal formulations prepared based on DOPE/CH/DSPE-MPEG.

\begin{tabular}{lccc}
\hline Target & Fluorescent dye & Fluorescent dye & Drug \\
\hline- & FITC & Alexa fluor 647 & MTX \\
SP-DS3 & FITC & Alexa fluor 647 & MTX \\
\hline
\end{tabular}




\section{Cell Culture Conditions}

Human peripheral blood mononuclear cells were isolated from buffy coats of blood donors from the Immunohaemotherapy Department of Hospital de São João (Porto, Portugal) by centrifugation over Lymphoprep (AxisShield, Norway), and monocytes were further purified by magnetic cell sorting using CD14 microbeads (Miltenyi Biotec, Germany). The ethics committee granted approval for the study. Monocyte-derived macrophages were generated in RPMI 1640 (supplemented with 10\% FCS, $100 \mathrm{U} / \mathrm{mL}$ penicillin and $100 \mu \mathrm{g} / \mathrm{mL}$ streptomycin) in the presence of $50 \mathrm{ng} / \mathrm{mL}$ M-CSF (PeproTech, UK) for 7 days followed by activation for $24 \mathrm{~h}$ with $20 \mathrm{ng} / \mathrm{mL}$ IL-4 (PeproTech, UK). Jurkat E6.1 T cells and THP-1 cells were routinely cultivated in RPMI 1640 medium supplemented with $10 \%$ FCS, $100 \mathrm{U} / \mathrm{mL}$ penicillin and $100 \mu \mathrm{g} / \mathrm{mL}$ streptomycin. Four days prior to the liposome uptake experiments, we transferred the cells to FAfree RPMI 1640 medium supplemented with $10 \%$ FCS, $100 \mathrm{U} / \mathrm{mL}$ penicillin and $100 \mu \mathrm{g} / \mathrm{mL}$ streptomycin. FR $\beta$ overexpressing THP-1 cells were prepared by retroviral transduction of full-length human FR $\beta$ using an in-house modified retroviral pBMN vector and subsequent FACS sorting to obtain a $99.9 \% \mathrm{FR} \beta$-positive population. THP-1 cells transduced with the empty vector were used as a control.

\section{Macrophage and T Cell Line Internalization}

Binding and internalization of liposomes by macrophages and $\mathrm{T}$ cells was assessed using a multi-spectral imaging flow cytometer. Briefly, cells were incubated for $1 \mathrm{~h}$ with $100 \mu \mathrm{g} / \mathrm{mL}$ liposomes in complete RPMI 1640 medium at $37^{\circ} \mathrm{C}$. After incubation, cells were washed twice with PBS (pH 7.4). Surface FR $\beta$ was stained using a monoclonal FR $\beta$ antibody (Exbio, Czech Republic) after blocking $\mathrm{Fc}$ receptors with FcR Blocking Reagent (Miltenyi Biotec, Germany). An isotype-matched monoclonal antibody was used as the negative control. Images of cells were acquired on a 6-channel ImageStreamX Imaging Flow Cytometer (Amnis, EMD Millipore) equipped with $488 \mathrm{~nm}$ and $785 \mathrm{~nm}$ excitation lasers, configured at $40 \times$ magnification and controlled by INSPIRE software. Imaging was performed at the Bioimaging Center for Biomaterials and Regenerative Therapies (b.IMAGE, INEB, Porto, Portugal). Approximately 20,000 events were collected per sample using a bright field (BF) area lower limit of $20 \mu \mathrm{m}^{2}$. Analysis was performed with IDEAS 5.0 (Amnis, EMD Millipore). The percentage of cells with internalized liposomes was determined using the Internalization Wizard application of INSPIRE. Briefly, after excluding out-of-focus images and selecting single cells, a gate was drawn on the liposome-positive cells, and a mask corresponding to the interior of the cell was created based on the BF image. Internalization was estimated by comparing the liposomal FITC fluorescence intensity inside the cell with the fluorescence intensity in the entire cell, and the percentage of cells with internalized liposomes was quantified. Liposomal uptake was also measured using a LSRII flow cytometer (BD) followed by analysis using FlowJo software (TreeStar).

\section{In Vivo Targeting of Liposomes to FR $\beta$ Murine Macrophages}

C57BL/6 mice were injected i.p. with zymosan (100 $\mu \mathrm{g}$, Sigma). Three days later, Alexa Fluor 647-labelled liposomes $(200 \mu \mathrm{l})$ were injected i.p., and peritoneal macrophages were harvested at different time points. Fc receptors were blocked by a $30 \mathrm{~min}$ incubation with normal goat and rabbit IgG $(10 \mu \mathrm{g} / \mathrm{ml})$ in PBS/1\% BSA. Cells were stained in the same medium with a murine $\operatorname{FR} \beta$ specific rat monoclonal antibody ${ }^{15}$ (kindly provided by Dr. T. Matsuyama (Department of Immunology, Graduate School of Medical and Dental Sciences, Kagoshima University, Japan) followed by a Dylight 488-conjugated antirat secondary antibody (eBioscience, USA). Cells were then stained for CD11b and F4/80 with CD11b-PE and F4/80-PerCP Cy5 conjugates (eBiosciences, USA). Flow cytometry data acquisition was performed using a FACS Calibur cytometer (Becton Dickinson) and analyzed with Kaluza software (Coulter).

\section{Collagen-Induced Arthritis}

Six-week-old DBA/1J Rj male mice were purchased from Janvier Laboratory (Le Genest-St-Isle, France). Mice were housed in groups of 6 per cage. Arthritis was induced with type II bovine collagen (CII) (MD Bioscience, Switzerland) as previously described. ${ }^{16,17}$ Male DBA/1 mice were injected subcutaneously at the base of the tail with $100 \mu \mathrm{g}$ of CII emulsified in Freund's adjuvant (BD DIFCO, Fisher Scientific, France). On day 21 postinjection, mice were boosted with a subcutaneous injection of CII in incomplete Freund's adjuvant (BD DIFCO, Fisher Scientific, Ilkirch, France). In this model, arthritis develops 20 to 30 days after the first collagen injection. Mice were monitored for evidence of arthritis in all 4 paws using a blind procedure. For each mouse, the clinical severity of arthritis was scored (0: normal; 1: erythema; 2: swelling; 3: deformity; and 4: ankylosis) in 10 joints or group of joints: 3 joints of the two hind legs (toes, tarsus, ankle). The maximum score reached for each of the 10 joints was 4 , and thus, the maximum clinical arthritis score for a single mouse on a given day was 40 . The mean arthritic score on each clinical observation day was calculated in each group. ${ }^{18}$ The various injection treatments were randomized in each cage to avoid cage dependency of the clinical score, and a trained operator for arthritis scoring (20 years) was blinded to the treatment injected in each mouse. Treatment started 14 days after immunization. The different formulations were injected twice per week i.p., and mice were scored on the same day. For 
liposome in vivo uptake analysis, Alexa Fluor 647 fluorescently labelled control or FA-SP-DS3 liposomes were injected i.v. into the tails of arthritic mice showing similar and high clinical scores $(>10)$. Liposome uptake in ankles was then monitored 30 min later by visualizing Alexa 647 fluorescence in living anesthetized animals using in vivo fluorescence imaging (Photon imager RT, Biospacelab). MTX was quantified after injection i.p. of $1.25 \mathrm{mg} / \mathrm{kg}$, and analysed via HPLCMSMS, (Agilent 1260 HPLC coupled to a Sciex API4000 device triple quadrupole mass spectrometer). MTX was detected in positive mode using the $+\mathrm{H}$ molecular ion. These studies were approved by the local animal experimentation ethics committee (CEEA no. 34, agreement no. CEEA34.GB.029.11).

\section{Statistical Analysis}

Statistical analyses were performed with GraphPad Prism software (version 5.0). Unless otherwise stated, differences were tested for statistical significance by a one-way ANOVA.).

\section{RESULTS}

\section{Cellular Uptake of Liposomes by FR-Expressing Macrophages}

We have previously described an innovative strategy for targeted liposomal delivery that uses a hydrophobic fragment of surfactant protein conjugated to a linker and FA, the SP-DS3 peptide, more efficient than the classic systems. ${ }^{10}$ This peptide was shown to insert deeply into the lipid bilayer without affecting the integrity of the liposomes ( 0.75 molar ratio). The combination of all complementary characteristics of these tailored liposomes, including their small size $(\sim 120 \mathrm{~nm})$, low cytotoxicity and their specific targeting of FR-expressing cells, ${ }^{10}$ supports their use as specific therapeutic nanodelivery systems as demonstrated here.

To evaluate the efficiency of FA targeting in liposomes integrating a peptide conjugated to a linker and FA (SP-DS3) we used activated human primary macrophages derived from human peripheral blood monocytes cultured in the presence of M-CSF because these cells express high levels of $\operatorname{FR} \beta$. Macrophages expressing FR $\beta$ are important cell targets for this type of delivery system as they display a circumscribed distribution in many inflammatory diseases and cancer. ${ }^{19,20}$ It has been reported that modifying the liposome surface with PEG greatly reduces the immune recognition of the liposomes and their subsequent clearance by the reticuloendothelial (mononuclear phagocyte) system. ${ }^{21,22}$ We therefore tested PEG at concentrations of $5 \%$ and $10 \%$ to improve the delivery specificity to activated FR $\beta$-expressing macrophages. PEG at $10 \%$ greatly improved the stealth degree of the liposomes, thereby reducing the non-specific uptake, and promoted the specificity of FA-mediated targeting (Fig. 1(A)). An increase in the percentage of SP-DS3 molecules, and consequently amount of FA on the liposomal surface, further improved this specific internalization (Fig. 1(A)). We also measured the uptake of liposomes with the improved PEG formulation in the monocytic cell line THP-1 with and without the overexpression of human $\mathrm{FR} \beta$. Compared to control liposomes, liposomes with FA were highly internalized in THP-1 cells retrovirally transformed with FR $\beta$ in comparison with the wild-type THP-1 cells that weakly express FR $\beta$ (data not shown) showing minimal uptake similar to the Jurkat $\mathrm{T}$ cells used as a negative control (Fig. 1(B)).

To test the specific targeting of activated macrophages in vivo, $\mathrm{FR} \beta$-expressing macrophages were elicited by intraperitoneal (i.p.) injection of zymosan into healthy mice. Three days later, these mice were injected i.p. with fluorescent liposomes for detailed uptake analysis. Only peritoneal cells showing increased expression of the CD11b and F4/80 macrophage markers expressed also high levels of FR $\beta$ and a very high uptake of fluorescent liposomes (Fig. 1(C)). Compared to control liposomes without FA, SP-DS3 liposomes exhibited high accumulation in this subset of cells (Fig. 1(D)).

In Vivo Targeting of Liposomes to Arthritic Joints To measure the in vivo uptake specificity of these new liposomes in a pathological context, arthritis was induced in DBA/1 mice using bovine type-II collagen (CIA model). Arthritic mice, showing similar and high clinical scores, were imaged after tail vein injection of FR-targeted and non-targeted liposomes filled with Alexa Fluor 647. Compared to liposomes without FA, SP-DS3 liposomes strongly accumulated in the joints of the arthritic mice (Fig. 2(A)). Of note in all mice a strong signal was also observed at the sites of injection of the liposomes in the tails. The mean fluorescence analysis in ankles of the two groups shows a significantly higher uptake of liposomesFA (data not shown). Furthermore, we also monitored the MTX concentration in the paws of CIA mice. MTX, firstline drug therapy for the treatment of RA, was encapsulated $(\sim 750 \mu \mathrm{g} / \mathrm{mL})$ in liposomes. We observed a higher level of MTX in the group treated with liposomes-FA, at peak and also during the whole kinetics, demonstrating the advantage of the SP-DS3 formulation.

We then measured the uptake of liposomes in cell populations from inflamed joints of arthritic mice. To address this point, we analyzed the cell populations within the ankles using CD11b, F4/80 and FR $\beta$ markers. Three main cell subsets can be clearly discriminated from CD11b and F4/80 labeling. Among these, the $C D 11 b^{\text {int }} / F 4 / 80^{\text {hi }}$ cell population showed the highest level of $\mathrm{FR} \beta$ expression (data not shown). By gating on these $\mathrm{FR} \beta+$ macrophages, we could not observe a higher percentage of Alexa Fluor-647 positive cells. However, by analyzing the cells expressing the highest levels of FR $\beta$, we observed that the 
A
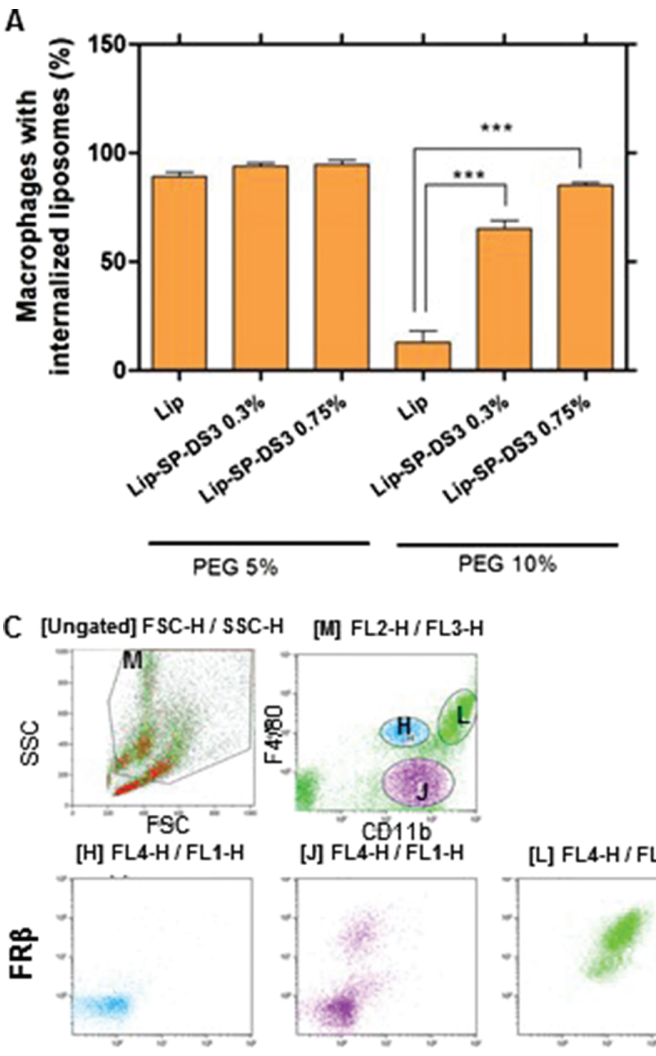
[J] FL4-H/FL1-H

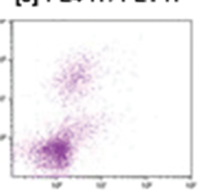
[L] FL4-H/FL1-H

Lip-SP-DS3

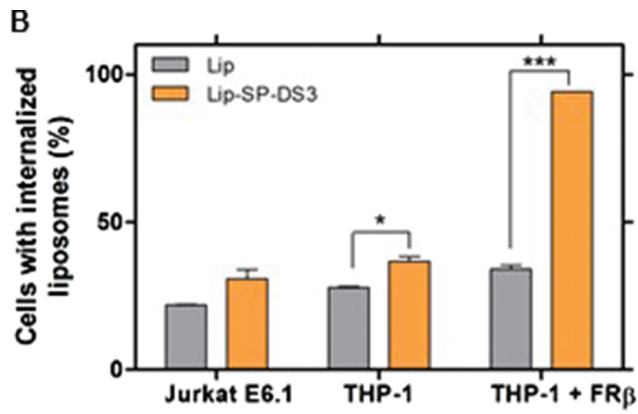

D

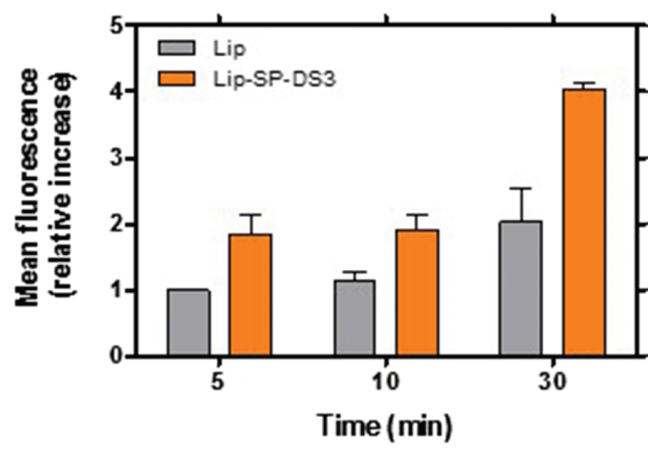

Figure 1. Specific uptake of liposomes by FR $\beta$-expressing cells. Specific uptake of liposomes by (A) FR $\beta$-expressing primary human macrophages $(1 \mathrm{~h})$ and $(\mathrm{B})$ monocytic THP-1 cell line over expressing human FR $\beta$ (24 h). (C) and (D) In vivo specific targeting of liposomes to FR $\beta$ murine peritoneal macrophages. Histograms in C show that only CD11 ${ }^{\text {hi }} F 4 / 80^{\text {hi }}$ macrophages ( $L$ population) have uptaken the fluorescently-labeled liposomes. Graphs in D represent the increase of fluorescence in these macrophages having internalized liposomes along time as determined by imaging flow cytometry analysis. Values are the mean $+\mathrm{SD}$ of 2 experiments.

number of macrophages having uptaken FA-liposomes was significantly higher in this cell subpopulation.

\section{In Vivo Clinical Assays}

In vivo evaluation of the clinical benefit of liposomal formulations encapsulating MTX administered i.p. was performed in arthritic mice, before disease onset. The results indicate that encapsulation of MTX in liposome preparations can improve prophylactic efficacy (Figs. 2(C) and (D)). The clinical benefit was significantly increased when using SP-DS3 liposomes displaying FA, where mice did not shown any clinical signs of disease, which is in good agreement with the specific targeting observed in vitro and the much stronger accumulation of MTX to the inflamed joints when it is delivered encapsulated into FA-containing liposomes. Comparatively, the drug injected in a soluble form, it only had a marginal effect and did not prevent the development of arthritis.

Studies have shown that cells of synovial fluid from arthritic joints express the ectonucleotidases CD39 and CD73, ${ }^{23,24}$ both required to hydrolyze ATP in adenosine and in turn limit inflammation. We therefore questioned the status of these ectonucleotidases in macrophage populations in joints of CIA mice treated or not with liposomes. To address this point ankles were digested in collagenase and cell populations analyzed using CD11b, F4/80, FR $\beta$, CD39 and CD73 markers. After a first gating on CD11b and F4/80 cells to select macrophages $\left(\mathrm{CD} 11 \mathrm{~b}^{\mathrm{int}} \mathrm{F} 4 / 80^{\mathrm{hi}}\right)$, a second gate on FR $\beta$ expression was defined (Fig. 3(A)). We found that expression of the ectonucleotidases CD73 and CD39 was decreased about 2-fold in this joint tissue macrophage population harvested from mice treated with FA-liposomes containing MTX compared with liposomes without FA and MTX (Figs. 3(B) and (C)). We observed intermediate levels of CD39 and CD73 in FA-liposomes treated mice, suggesting an effect of the liposomes just by targeting the FR $\beta$. We also showed that the expression of FR $\beta$ also tends to decrease similarly (Fig. 3(D)).

CD39 and CD73 ectonucleotidase expression levels were also analyzed in relation to $\mathrm{FR} \beta$ expression. We observed that their expression correlated with FR $\beta$ expression levels (Figs. 4(A) and (B)). The expression levels of CD39 and CD73 were also correlated to the clinical score for each posterior leg or to the total clinical score, the 

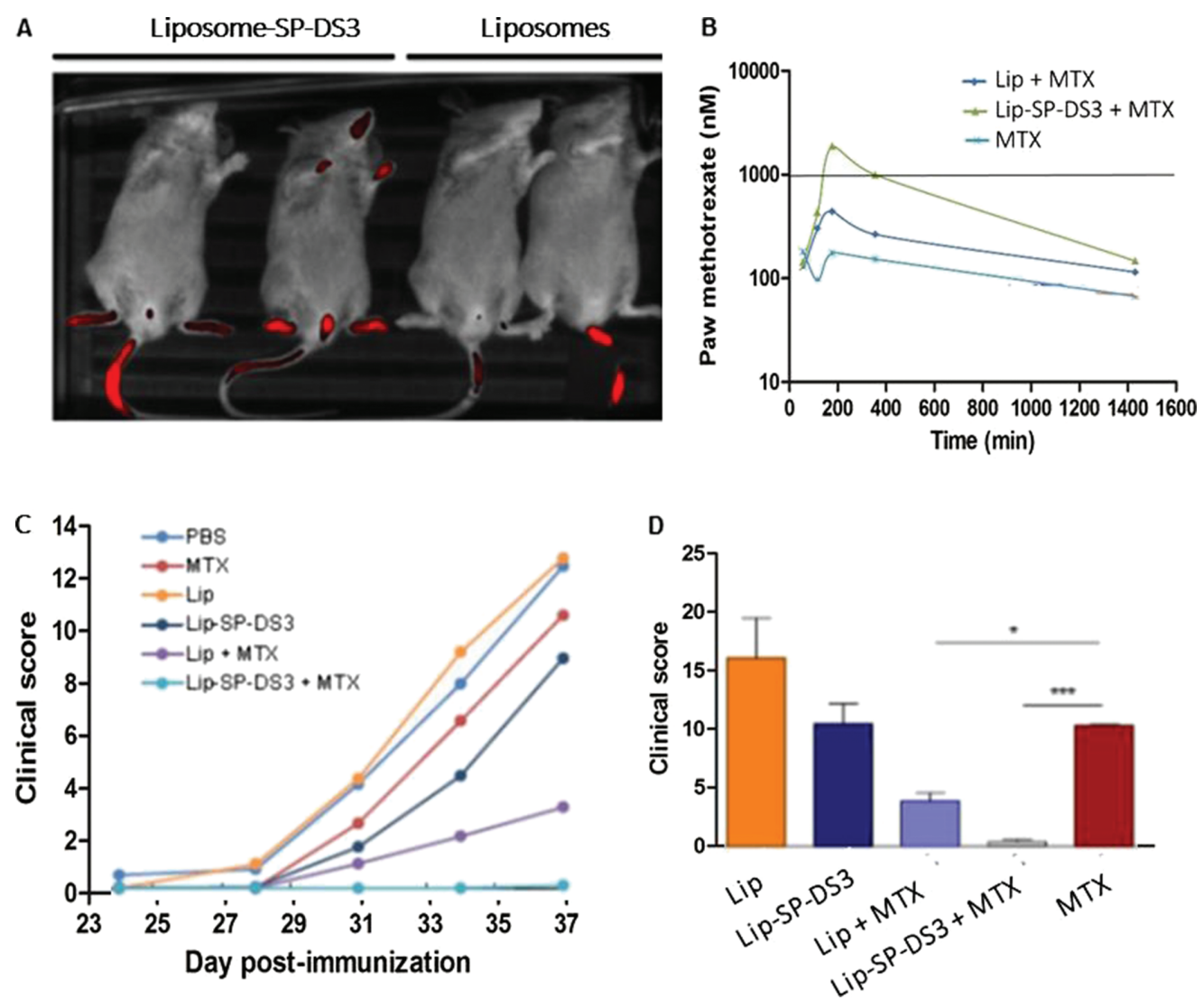

Figure 2. In vivo specific targeting and prophylactic efficiency of FR-targeted liposomes in arthritic mice. In vivo uptake specificity of (A) Alexa Fluor 647 fluorescently labelled liposomes $(30 \mathrm{~min})$ and (B) liposomes encapsulating MTX (following i.p. application of $1.25 \mathrm{mg} / \mathrm{kg}$ MTX). (C) Clinical effects of liposomes encapsulating MTX on arthritis. The mean clinical score in each group (12 mice/group) over time is shown. A Dunn's multiple comparison test was performed $(P$ value $<0.05)$. (D) Mean clinical scores + SD from two independent experiments performed as in (C) are shown. Unpaired two-tailed $t$ test was performed $(P$ value $=0.006)$.

latter taking into account clinical scores of both posterior and anterior legs. As shown in Figures 5(A) and (B), high clinical scores translate directly into higher levels of CD73, when either leg or total clinical scores are considered. The same observation was made concerning CD39 (Figs. 5(C) and S(D)). Importantly, treatment of mice with FA-liposomes led to an intermediary total clinical score that accords to an intermediary average level of CD39 or CD73 (Figs. 5(B) and (D)).

\section{DISCUSSION}

The drugs presently used in RA treatment are often ineffective and frequently present serious side effects that can even lead to the death of the patient. Despite their high clinical effectiveness, the cost efficacy of biologicals agents, namely anti-TNF- $\alpha$ molecules, is questionable. Therefore it is imperative the development of alternative, cost efficient treatment for RA, with low side effects, that will constitute a more advantageous solution than current therapies. In earlier studies of RA, in inflamed joints, a subpopulation of activated macrophages was observed which also expresses a receptor for the FA vitamin. Because no other tissues/cell types expressed the $\operatorname{FR} \beta$ (except the kidneys proximal tubules and placenta and in normal hematopoietic tissue), accumulation of FR-macrophages in arthritic joints allowed the selective targeting of FA linked imaging and therapeutic agents to these sites of inflammation. ${ }^{25}$ FA-targeted therapies, selectively attack the pathologic cell type, leaving the vast majority of macrophages unharmed. As a consequence, the level of toxicity associated with FAtargeted therapy appears to be very low. ${ }^{11}$ Recently, Low and colleagues developed an antibody with high specificity towards human $\operatorname{FR} \beta$ and demonstrated that this antibody is able to target $\operatorname{FR} \beta$ positive cells including macrophages from RA patients. ${ }^{26}$ Likewise, other efforts in therapy include dsFv anti-FR $\beta$-targeted Pseudomonas exotoxin $\mathrm{A},{ }^{27} \mathrm{FA}$ hapten-mediated immunotherapies ${ }^{28,29}$ and anti-FA designed to bind FR. ${ }^{30}$ These findings suggest that $\mathrm{FR} \beta$ therapies can be applied as a research tool for effective targeting of activated macrophages during inflammatory disease progression. This observation, together with the observation that arthritic joints are readily visualized with FA-targeted radiopharmaceuticals in 
A

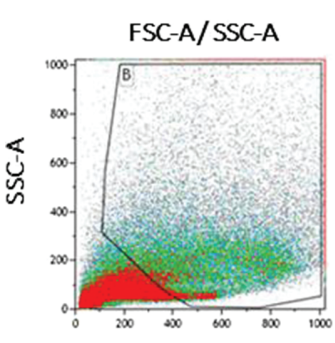

FSC-A

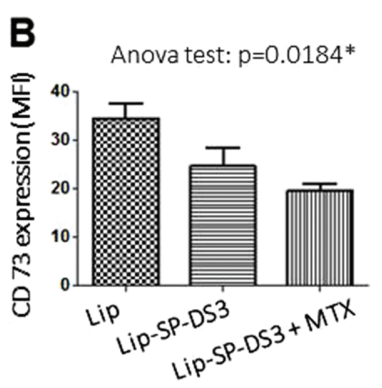

C

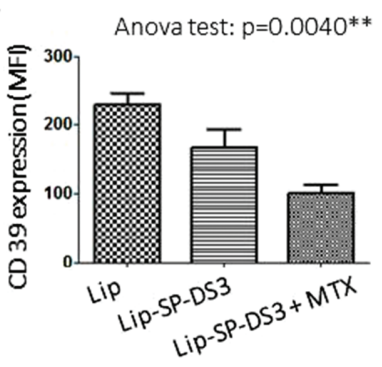

D

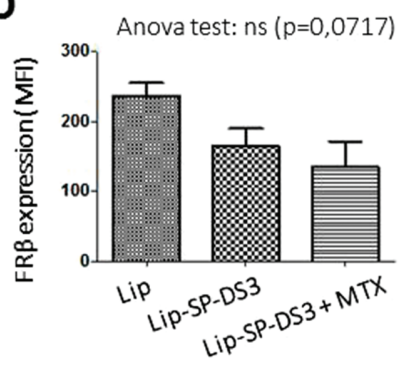

Figure 3. CD73, CD39 and FR $\beta$ expression by joint tissue macrophages depending on treatment of CIA mice with liposomes. (A) Gating strategy. Ankles were removed from CIA mice which received liposomes. Cells were stained with anti-CD11b, anti-F4/80, anti-CD73, anti-CD39 and FR $\beta$ antibodies. Expression of CD73, CD39 and FR $\beta$ was analyzed on $C D 11 b^{\text {int }} / F 4 / 80^{\text {hi }}$ macrophage cell population (gate C). Mean fluorescence intensities of CD 73 (B), CD39 (C) and FR $\beta$ (D) in gate C after the different treatments are shown. Statistical values (Student's test analysis) are also shown.

patients with RA $\left({ }^{18} \mathrm{~F}\right.$-polyethylene, PET tracer ${ }^{31}$ and glycol-folate ${ }^{99 \mathrm{~m}} \mathrm{Tc}-\mathrm{EC} 20$, FolateScan ${ }^{32}$ ), promises well for a successful response to FA-targeted immunotherapy in humans. Although each of the aforementioned approaches holds promise for yielding new therapeutic options for patients, there have been no reports for FR-mediated targeting anti-inflammatory drugs to sites of inflammation, encapsulated in drug delivery systems. Recently, Thomas T. and colleagues developed a FA-conjugated dendrimers to target macrophages in inflammatory disease of arthritis. ${ }^{33}$ However, the surface conjugation of de drug MTX prevents their protection from degradation or deactivation prior to reaching target site in vivo. Liposomes have gained extensive attention as carriers for a wide range of drugs due being both nontoxic and biodegradable because they are composed of naturally occurring substances. ${ }^{34,35}$ Biologically active materials encapsulated within liposomes are protected to varying extent from immediate
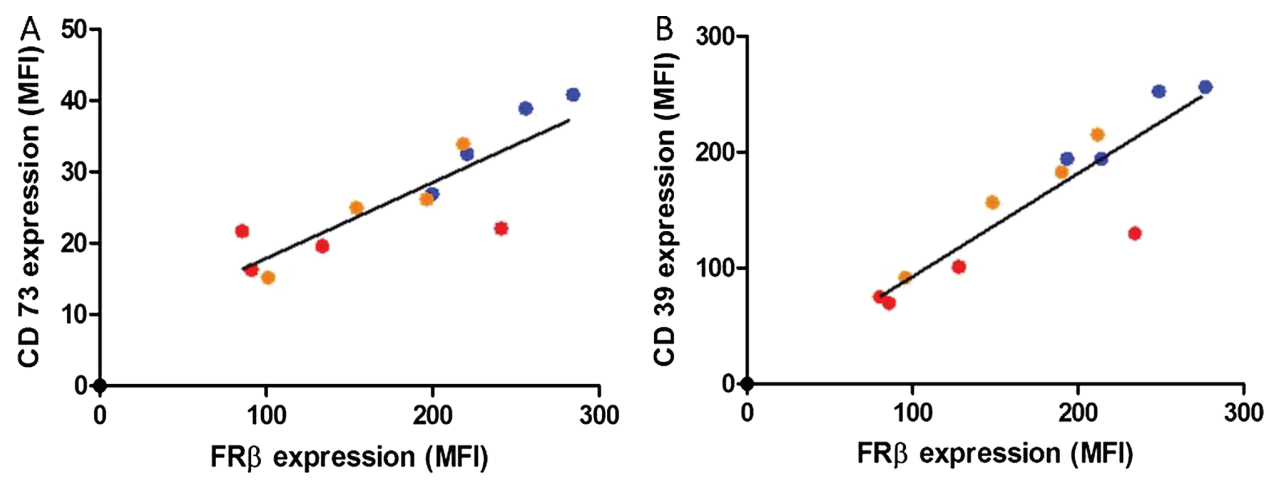

- LLip-SP-DS3 - LLP-SP-DS3+MTX

Figure 4. Correlation of CD73 (A) and CD39 (B) expression levels with FR $\beta$ expression by joint tissue macrophages depending on treatment of CIA mice with liposomes. Ankles were removed from CIA mice which received liposomes. CD73, CD39 and FR $\beta$ membrane levels were measured by FACS as described in Figure 3. Each dot represents one ankle from a separate mouse. 

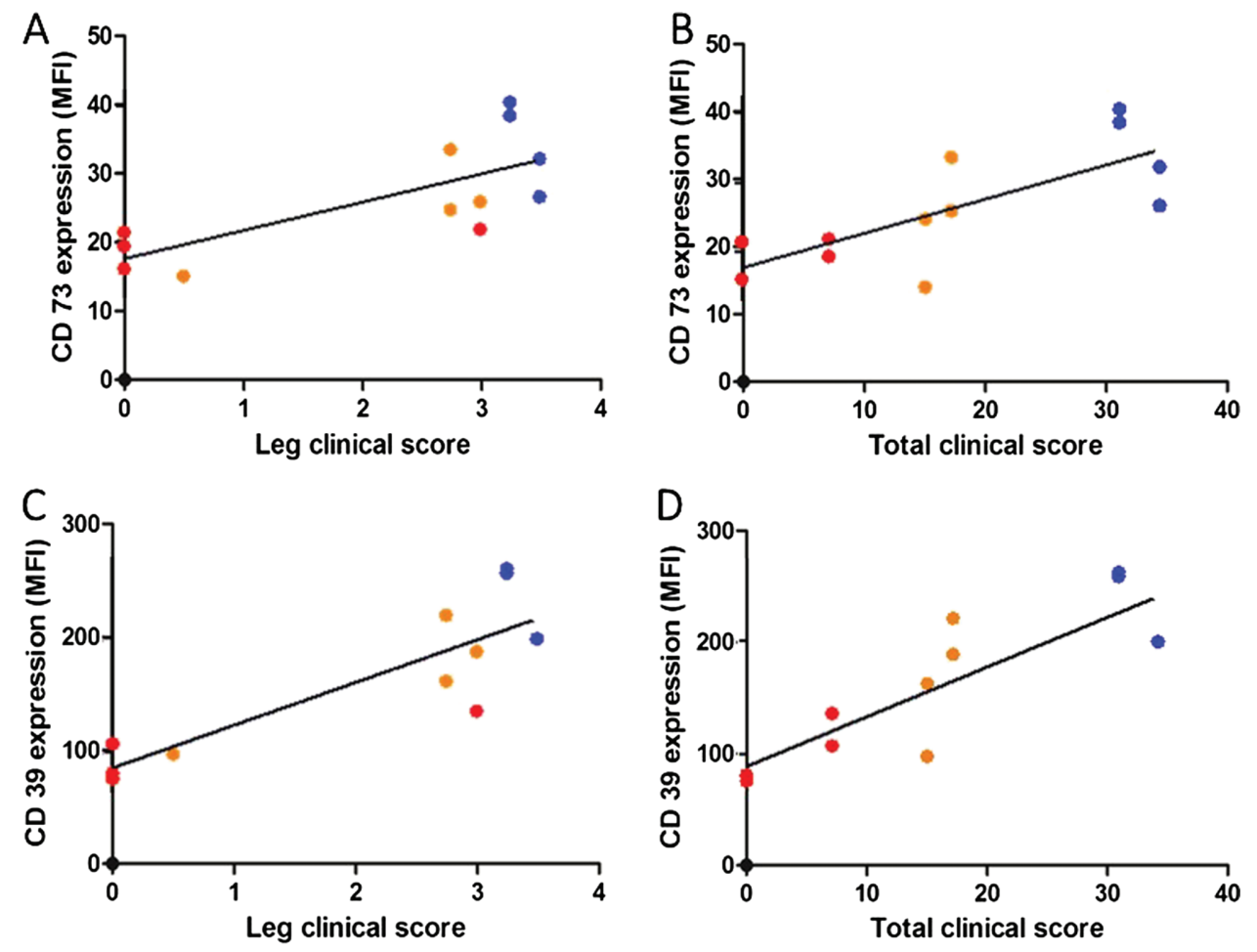

Lip Lip-SP-DS3 Lip-SP-DS3 + MTX

Figure 5. CD39 and CD73 macrophage expressions correlate with clinical scores. CD73 and CD39 expressions of $\mathrm{CD} 11 \mathrm{~b}^{+ \text {int }} \mathrm{F} 4 / 80^{\mathrm{hi}}$ macrophages harvested from ankles used in Figure 4 were correlated with the clinical score of each joint ( $A$ and $C$, respectively) or the total clinical scores (B and D, respectively).

dilution or degradation, suggesting drug carrier systems for the transport of drugs and other bioactive capsules to disease affected organs. ${ }^{36-38}$ We have developed a novel FR-targeted liposomes by incorporating a bifunctional SPDS3 peptide consisting of the neck domain of surfactant proteins, which serves both as a linker and an anchor of the FA to the liposomal membrane. This delivery system proved to be more efficient than classic systems where the FA is linked to liposomes by PEG. The combination of all complementary characteristics of these tailored liposomes, including their small size, lack of cytotoxicity and their specific targeting of FR $\alpha$-expressing cells, supports their use as specific therapeutic nanodelivery systems as demonstrated here by the biological effect of several drugs. The exciting results obtained with $\mathrm{FR} \alpha$ cancer cells led us to investigate their use in $\mathrm{FR} \beta$ expressing cells, namely activated macrophages, the key effector cells in RA. The inclusion of PEG in liposomal bilayers has extended blood circulation time of conventional liposomes and is considered as a major breakthrough in liposome mediated drug delivery. ${ }^{22}$ The results show that the presence of $5 \%$ PEG was insufficient to reduce the internalization of liposomes by activated human primary macrophages. ${ }^{21}$ The increase to $10 \%$ PEG greatly improved the stealth degree of the liposomes, thereby reducing the non-specific uptake, and promoted the specificity of FA-mediated targeting. The low internalization of FA-bearing liposomes in cells with negligible FR expression (THP-1 macrophages and Jurkat $\mathrm{T}$ cells) compared to macrophages overexpressing FR $\beta$ (THP-1 retrovirally transformed to overexpress FR $\beta$ ) demonstrated that their uptake by immune cells is dependent on FR $\beta$ expression. Furthermore, the results obtained in activated macrophages in vivo clearly demonstrate the potential of these novel liposomes that integrate SP-DS3 to target $\mathrm{FR} \beta$-expressing macrophages.

To test the specificity of these new liposomes in a pathological context, arthritis was induced in mice (CIA model), and the results showed that liposomes strongly accumulated in their joints. Furthermore, the analysis of cell populations from inflamed joints of arthritic mice revealed that macrophages expressing high levels of FR $\beta$ are more prone to uptake FR-targeted than the non-targeted liposomes.

To prove the ability of our liposomal formulation as drug delivery system, liposomes encapsulating MTX were administered in arthritic mice, before disease onset. Complete prophylactic efficacy was observed in mice treated with SP-DS3 liposomes encapsulating MTX, where mice did not shown any clinical signs of arthritis. Comparatively, when the drug was injected in a soluble form it only had a marginal effect and did not prevent the development of arthritis. This fact lead us to believe that encapsulation of MTX in our proposed formulation offers a cost effective 
way to treat arthritis and delay or reduce MTX intolerance. The results presented here might have an important implication in clinical practice, where available biological therapies could be delayed or completely replaced by the proposed MTX liposomal formulations tested in this work. It brings new hope to a large number of patients who become intolerant to MTX and require much more expensive treatments with biological agents.

It was found that the ectonucleotidases CD39 and CD73, required to hydrolyze ATP in adenosine and in turn limit inflammation, are express in cells of synovial fluid from arthritic joints. In good agreement with this observation, our results show that the presence of $\mathrm{CD} 39+\mathrm{CD} 73+$ $\mathrm{FR} \beta+$ expressing macrophages is correlated with a high clinical score. Moreover, this cell population is less abundant in arthritic mice treated with liposomes containing FA, especially when MTX was encapsulated in the device. Thus, efficiency of our liposomal formulation in RA treatment might be the direct consequence of this regulation of CD39 and CD73 ectonucleotidase expression by macrophages.

In conclusion, the use of liposomes equipped with the novel bifunctional SP-DS3 peptide linker for FA-mediated delivery opens new opportunities for the cost-effective treatment of human chronic inflammatory diseases as RA.

Acknowledgments: Eugénia Nogueira (SFRH/BD/ 81269/2011), Ana Loureiro (SFRH/BD/81479/2011) and Catarina Almeida (SFRH/BPD/48533/2008) hold scholarships from Fundação para a Ciência e a Tecnologia (FCT). Gonçalo J. L. Bernardes is a Royal Society University Research Fellow at the Department of Chemistry, University of Cambridge and an Investigador FCT at the Instituto de Medicina Molecular, Faculdade de Medicina da Universidade de Lisboa. This study was funded by the European Union Seventh Framework Programme (FP7/2007-2013) under grant agreement NMP4-LA-2009228827 NANOFOL. This study was also supported by FEDER through POFC-COMPETE, by national funds from FCT through the project PEst-C/BIA/UI4050/2014 and the strategic funding of ID/BIO/04469/2013 unit. We thank the Immuno-haemotherapy Department of Hospital de São João (Porto, Portugal) for providing buffy coats from healthy volunteers. We also thank Noëmy Gueriba for her technical assistance in various experiments.

\section{REFERENCES}

1. G. S. Firestein, Evolving concepts of rheumatoid arthritis. Nature 423, 356 (2003).

2. J. Presumey, G. Courties, P. Louis-Plence, V. Escriou, D. Scherman, Y. M. Pers, H. Yssel, J. Pene, D. Kyburz, S. Gay, C. Jorgensen, and F. Apparailly, Nicotinamide phosphoribosyltransferase/visfatin expression by inflammatory monocytes mediates arthritis pathogenesis. Ann. Rheum. Dis. 72,1717 (2013).

3. F. M. Brennan and I. B. McInnes, Evidence that cytokines play a role in rheumatoid arthritis. J. Clin. Invest. 118, 3537 (2008).
4. J. S. Smolen, R. Landewé, F. C. Breedveld, M. Buch, G. Burmester, M. Dougados, P. Emery, C. Gaujoux-Viala, L. Gossec, J. Nam, S. Ramiro, K. Winthrop, M. de Wit, D. Aletaha, N. Betteridge, J. W. J. Bijlsma, M. Boers, F. Buttgereit, B. Combe, M. Cutolo, N. Damjanov, J. M. W. Hazes, M. Kouloumas, T. K. Kvien, X. Mariette, K. Pavelka, P. L. C. M. van Riel, A. Rubbert-Roth, M. Scholte-Voshaar, D. L. Scott, T. Sokka-Isler, J. B. Wong, and D. van der Heijde, EULAR recommendations for the management of rheumatoid arthritis with synthetic and biological disease-modifying antirheumatic drugs: 2013 update. Ann. Rheum. Dis. 73, 492 (2013).

5. L. D. Quan, G. M. Thiele, J. Tian, and D. Wang, The development of novel therapies for rheumatoid arthritis. Expert Opin. Ther. Pat. 18, 723 (2008).

6. M. Bulatović, M. W. Heijstek, M. Verkaaik, E. H. P. van Dijkhuizen, W. Armbrust, E. P. A. Hoppenreijs, S. Kamphuis, W. Kuis, T. C. G. Egberts, G. Sinnema, C. M. A. Rademaker, and N. M. Wulffraat, High prevalence of methotrexate intolerance in juvenile idiopathic arthritis: Development and validation of a methotrexate intolerance severity score. Arthritis Rheum. 63, 2007 (2011).

7. I. Colmegna, B. R. Ohata, and H. A. Menard, Current understanding of rheumatoid arthritis therapy. Clin. Pharmacol. Ther. 91, 607 (2012).

8. J. M. Kremer, G. S. Alarcón, R. W. Lightfoot, R. F. Willkens, D. E. Furst, H. J. Williams, P. B. Dent, and M. E. Weinblatt, Methotrexate for rheumatoid arthritis. Arthritis Rheum. 37, 316 (1994).

9. S. Merkesdal, J. Ruof, T. Mittendorf, and H. Zeidler, Costeffectiveness of TNF- $\alpha$-blocking agents in the treatment of rheumatoid arthritis. Expert. Opin. Pharmacother. 5, 1881 (2004).

10. E. Nogueira, I. C. Mangialavori, A. Loureiro, N. G. Azoia, M. P. Sárria, J. Härmark, U. Shimanovich, A. Rollett, G. J. L. Bernardes, G. Guebitz, H. Hebert, J. P. F. C. Rossi, A. C. Gomes, A. Preto, and A. Cavaco-Paulo, Folate-tailed peptide from surfactant protein D enhances liposome drug delivery. (2015), submitted.

11. C. M. Paulos, M. J. Turk, G. J. Breur, and P. S. Low, Folate receptormediated targeting of therapeutic and imaging agents to activated macrophages in rheumatoid arthritis. Adv. Drug Deliv. Rev. 56, 1205 (2004).

12. I.-M. Jou, C.-F. Lin, K.-J. Tsai, and S.-J. Wei, Macrophage-mediated inflammatory disorders. Mediators Inflamm. 2013, 3 (2013).

13. B. Varghese, N. Haase, and P. S. Low, Depletion of folatereceptor-positive macrophages leads to alleviation of symptoms and prolonged survival in two murine models of systemic lupus erythematosus. Mol. Pharm. 4, 679 (2007).

14. P. S. Low, W. A. Henne, and D. D. Doorneweerd, Discovery and development of folic-acid-based receptor targeting for imaging and therapy of cancer and inflammatory diseases. Acc. Chem. Res. 41, 120 (2007).

15. T. Nagai, M. Tanaka, Y. Tsuneyoshi, B. Xu, S. Michie, K. Hasui, H. Hirano, K. Arita, and T. Matsuyama, Targeting tumor-associated macrophages in an experimental glioma model with a recombinant immunotoxin to folate receptor $\beta$. Cancer Immunol. Immunother. 58, 1577 (2009).

16. J. S. Courtenay, M. J. Dallman, A. D. Dayan, A. Martin, and B. Mosedale, Immunisation against heterologous type II collagen induces arthritis in mice. Nature 283, 666 (1980).

17. N. Saidenberg-Kermanac'h, N. Bessis, D. Lemeiter, M. C. de Vernejoul, M. C. Boissier, and M. Cohen-Solal, Interleukin-4 cellular gene therapy and osteoprotegerin decrease inflammation-associated bone resorption in collagen-induced arthritis. J. Clin. Immunol. 24, 370 (2004).

18. G. Clavel, C. Marchiol-Fournigault, G. Renault, M.-C. Boissier, D. Fradelizi, and N. Bessis, Ultrasound and Doppler micro-imaging in a model of rheumatoid arthritis in mice. Ann. Rheum. Dis. 67, 1765 (2008).

19. A. Puig-Kröger, E. Sierra-Filardi, A. Domínguez-Soto, R. Samaniego, M. T. Corcuera, F. Gómez-Aguado, M. Ratnam, P. Sánchez-Mateos, and A. L. Corbí, Folate receptor $\beta$ is expressed 
by tumor-associated macrophages and constitutes a marker for M2 anti-inflammatory/regulatory macrophages. Cancer Res. 69, 9395 (2009).

20. J. W. Van Der Heijden, R. Oerlemans, B. A. C. Dijkmans, H. Qi, C. J. V. D. Laken, W. F. Lems, A. L. Jackman, M. C. Kraan, P. P. Tak, M. Ratnam, and G. Jansen, Folate receptor $\beta$ as a potential delivery route for novel folate antagonists to macrophages in the synovial tissue of rheumatoid arthritis patients. Arthritis Rheum. 60, 12 (2009).

21. E. Nogueira, A. Loureiro, P. Nogueira, J. Freitas, C. R. Almeida, J. Harmark, H. Hebert, A. Moreira, A. M. Carmo, A. Preto, A. C. Gomes, and A. Cavaco-Paulo, Liposome and protein based stealth nanoparticles. Faraday Discuss 166, 417 (2013).

22. A. L. de Barros, L. Mota, D. C. Soares, C. M. de Souza, G. D. Cassali, M. C. Oliveira, and V. N. Cardoso, Long-circulating, pHsensitive liposomes versus long-circulating, non-pH-sensitive liposomes as a delivery system for tumor identification. J. Biomed. Nanotechnol. 9, 1636 (2013).

23. B. Csóka, Z. Selmeczy, B. Koscsó, Z. H. Németh, P. Pacher, P. J. Murray, D. Kepka-Lenhart, S. M. Morris, W. C. Gause, S. J. Leibovich, and G. Haskó, Adenosine promotes alternative macrophage activation via $\mathrm{A} 2 \mathrm{~A}$ and $\mathrm{A} 2 \mathrm{~B}$ receptors. FASEB $J$. 26, 376 (2012).

24. L. Antonioli, P. Pacher, E. S. Vizi, and G. Hasko, CD39 and CD73 in immunity and inflammation. Trends Mol. Med. 19, 355 (2013).

25. W. Xia, A. R. Hilgenbrink, E. L. Matteson, M. B. Lockwood, J. X. Cheng, and P. S. Low, A functional folate receptor is induced during macrophage activation and can be used to target drugs to activated macrophages. Blood 113, 438 (2009).

26. Y. Feng, J. Shen, E. D. Streaker, M. Lockwood, Z. Zhu, P. S. Low, and D. S. Dimitrov, A folate receptor beta-specific human monoclonal antibody recognizes activated macrophage of rheumatoid patients and mediates antibody-dependent cell-mediated cytotoxicity. Arthritis Res. Ther. 13, 1 (2011).

27. R. Nagayoshi, T. Nagai, K. Matsushita, K. Sato, N. Sunahara, T. Matsuda, T. Nakamura, S. Komiya, M. Onda, and T. Matsuyama, Effectiveness of anti-folate receptor beta antibody conjugated with truncated pseudomonas exotoxin in the targeting of rheumatoid arthritis synovial macrophages. Arthritis Rheum. 52, 2666 (2005).

28. C. M. Paulos, B. Varghese, W. R. Widmer, G. J. Breur, E. Vlashi, and P. S. Low, Folate-targeted immunotherapy effectively treats established adjuvant and collagen-induced arthritis. Arthritis Res. Ther. 8, 28 (2006).
29. Y. S. Yi, W. Ayala-Lopez, S. A. Kularatne, and P. S. Low, Folatetargeted hapten immunotherapy of adjuvant-induced arthritis: Comparison of hapten potencies. Mol. Pharm. 6, 1228 (2009).

30. J. W. van der Heijden, R. Oerlemans, B. A. Dijkmans, H. Qi, C. J. van der Laken, W. F. Lems, A. L. Jackman, M. C. Kraan, P. P. Tak, M. Ratnam, and G. Jansen, Folate receptor beta as a potential delivery route for novel folate antagonists to macrophages in the synovial tissue of rheumatoid arthritis patients. Arthritis Rheum. 60, 12 (2009).

31. Y. Y. Gent, K. Weijers, C. F. Molthoff, A. D. Windhorst, M. C. Huisman, D. E. Smith, S. A. Kularatne, G. Jansen, P. S. Low, A. A. Lammertsma, and C. J. van der Laken, Evaluation of the novel folate receptor ligand $[18 \mathrm{~F}]$ fluoro-PEG-folate for macrophage targeting in a rat model of arthritis. Arthritis Res. Ther. 15, 1 (2013).

32. E. L. Matteson, V. J. Lowe, F. G. Prendergast, C. S. Crowson, K. G. Moder, D. E. Morgenstern, R. A. Messmann, and P. S. Low, Assessment of disease activity in rheumatoid arthritis using a novel folate targeted radiopharmaceutical Folatescan. Clin. Exp. Rheumatol. 27,253 (2009).

33. T. P. Thomas, S. N. Goonewardena, I. J. Majoros, A. Kotlyar, Z. Cao, P. R. Leroueil, and J. R. Baker, Jr., Folate-targeted nanoparticles show efficacy in the treatment of inflammatory arthritis. Arthritis Rheum. 63, 2671 (2011).

34. M. Li, H. Deng, H. Peng, and Q. Wang, Functional nanoparticles in targeting glioma diagnosis and therapies. J. Nanosci. Nanotechnol. 14, 415 (2014).

35. F. Jia, X. Liu, L. Li, S. Mallapragada, B. Narasimhan, and Q. Wang, Multifunctional nanoparticles for targeted delivery of immune activating and cancer therapeutic agents. J. Control. Release 172, 1020 (2013).

36. R. M. Lopes, M. M. Gaspar, J. Pereira, C. V. Eleutério, M. Carvalheiro, A. J. Almeida, and M. E. M. Cruz, Liposomes versus lipid nanoparticles: Comparative study of lipid-based systems as oryzalin carriers for the treatment of leishmaniasis. J. Biomed. Nanotechnol. 10, 3647 (2014).

37. W. Yuan, R. Kuai, R. Ran, L. Fu, Y. Yang, Y. Qin, Y. Liu, J. Tang, H. Fu, Q. Zhang, M. Yuan, Z. Zhang, F. Gao, and Q. He, Increased delivery of doxorubicin into tumor cells using extracellularly activated TAT functionalized liposomes: In vitro and in vivo study. J. Biomed. Nanotechnol. 10, 1563 (2014).

38. M. Liu, M. Li, G. Wang, X. Liu, D. Liu, H. Peng, and Q. Wang, Heart-targeted nanoscale drug delivery systems. J. Biomed. Nanotechnol. 10, 2038 (2014). 